\title{
An Adaptive Filtering Method for HRG Random Error in case of Colored Noise
}

\author{
Haotian Yanga, ${ }^{\text {, }}$, Lixin Wang ${ }^{b}$ and Zhuo Lic \\ Xi'an High-Tech Research Institute, Xi'an 710025, China \\ a Corresponding author: 745763540@qq.com, b15191918796@163.com, c18392432652@163.com
}

Keywords: ARMA model; orthogonal projection theorem; adaptive filtering.

\begin{abstract}
ARMA modeling and filtering methods for HRG random error are studied. Firstly, the model is constructed by analyzing the autocorrelation and partial correlation of HRG stationary random signals, the applicable model is build, and then the model order is estimated. Then, the residual parameters are obtained by calculating the residual method by long autoregressive model. Under the condition of colored noise, the ARMA model cannot be whitened by the traditional state expansion method. According to the equivalence between the orthogonal projection theorem and the linear minimum variance estimation, the adaptive kalman filter formula with the control term is deduced. The new method can directly reflect the influence of colored noise on the system, which can effectively eliminate the error and get the accurate estimation of the state value. The experimental results show that the improved method is more effective than the classical kalman filtering method in filtering out the noise.
\end{abstract}

\section{Introduction}

As an important inertial sensitive device, Gyroscope's accuracy affects the navigation system navigation performance at a large extent. In general, the gyroscope error mainly includes deterministic and random errors, the former can be compensated through the calibration method; the latter needs to be modeled filter compensation ${ }^{[1]}$.

At present, the more commonly used gyroscope random error modeling methods are: time series analysis[2-4], Neural network[5], Non-linear modeling[6] etc.Among them, because the noise in ARMA model is colored noise, it can not be whitened by traditional state expansion method, and can not directly use kalman filter, which makes the research based on ARMA model and kalman filter method still need further study. In response to this problem, many scholars have also put forward some solutions.In [7], the Kalman filter method with colored noise is deduced by using the linear variation, considering the correlation property of the adjacent epochs of colored noise;In [8], singular value decomposition and measurement transform method are used to propose matrix-weighted fusion kalman filtering method; In [9], the random noise based on ARMA model is modeled and filtered.Through the analysis of HRG stationary random data, it is considered that ARMA modeling is more suitable. When combined with Kalman filter technology, the problem of colored noise whitening should be considered. In this paper, the adaptive Kalman filter equation of colored noise is deduced by orthogonal projection correlation property analysis. The research of adaptive kalman filtering method based on ARMA model is carried out.

\section{Data Preprocessing and Correlation Test}

In the time series modeling process, the data should satisfy the condition of smooth, normal and zero mean. Therefore, after obtaining the random error of the gyroscope, the data must be checked to meet the modeling requirements ${ }^{[10]}$. After a first-order difference, a domestic HRG gets a smooth random data through static experiments, as shown in Figure 1. 


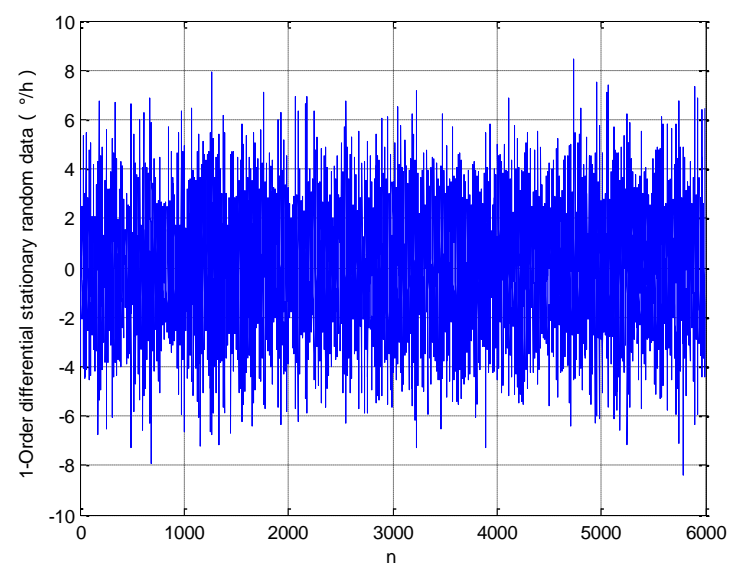

Figure 1. 1-Order differential stationary random data of HRG gyro

Through the analysis of auto-correlation and partial correlation of stationary random data, as shown in Fig. 2 and Fig.3. it is shown that the auto-correlation and partial correlation properties are trailing characteristics, and the data is more suitable ARMA model according to the time modeling theory[11-12].

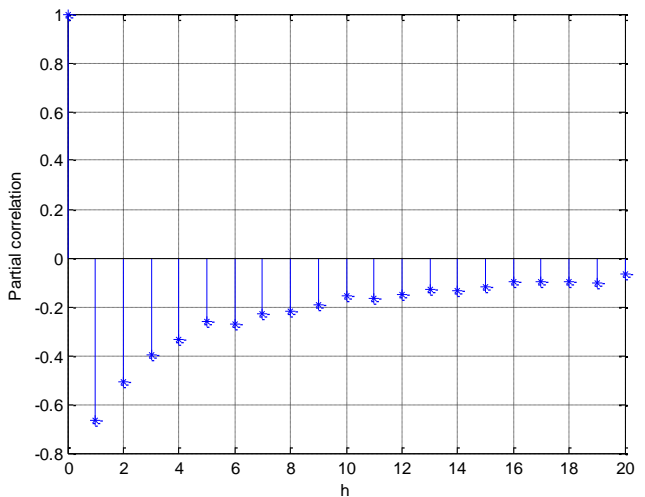

Figure 2. Partial correlation of stationary random data

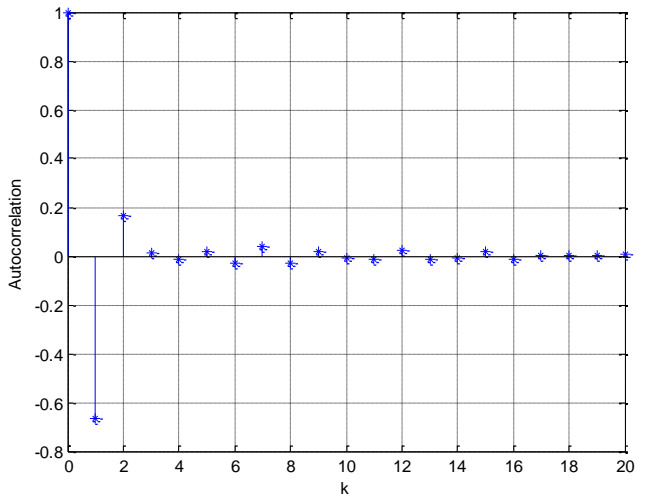

Figure 3. Autocorrelation of stationary random data

The ARMA model can be defined as[12]:

$$
x(t)=\sum_{i=1}^{n} a_{i} x(k-i)+\varepsilon(k)-\sum_{j=1}^{m} b_{i} n(k-j)
$$

Where $n$ is the order of the AR model, $m$ is the order of the MA model, $x(t) \mathrm{x}$ is the time series signal, $\varepsilon(k)$ is the white noise sequence, $a_{i}$ is the autoregressive coefficient and $b_{i}$ is the sliding coefficient.

In the ARMA model, the computational complexity of the determinant method is large, and the Ftest method leads to the uncertainty of the model due to the significant level. In order to ensure the accuracy of the model, the AIC (Akaike Information Criterion) criterion and the Bayesian Information Criterion (BIC) criterion are used to determine the model order ${ }^{[13]}$. The order of the ARMA model is $n=3, m=1$, Since the residual $\hat{\varepsilon}(k)$ of the ARMA model is unknown and the model 
parameters can not be estimated linearly, the residual equation is calculated by using the long autoregressive model to estimate the model parameters of HRG random error. In order to estimate the model parameters $a_{i}, b_{j}$ by using the least squares method, a higher order model $\operatorname{AR}(6)$ is used to approximate ARMA $(3,1)$. The residual estimate sequence at this time is the estimate of the residuals $\hat{\varepsilon}(k)$ of the ARMA model. Firstly, the AR(6) model is established :

$$
\begin{aligned}
x(k)= & -1.502 x(k-1)-1.609 x(k-2)-1.423 x(k-3)-1.071 x(k-4) \\
& -0.6474 x(k-5)-0.269 x(k-6)+\varepsilon(k)
\end{aligned}
$$

The estimated value $\hat{\varepsilon}(k)$ of the driving white noise can be derived from the above equation:

$$
\begin{aligned}
\hat{\varepsilon}(k) & =x(k)+1.502 x(k-1)+1.609 x(k-2)+1.423 x(k-3)+1.071 x(k-4) \\
& +0.6474 x(k-5)+0.269 x(k-6)
\end{aligned}
$$

The ARMA $(3,1)$ model can be obtained by using the recursive least squares method:

$$
\begin{aligned}
x(k)= & -0.5671 x(k-1)-0.2613 x(k-2)-0.0697 x(k-3) \\
& -0.9963 \varepsilon(k-1)+\varepsilon(k)
\end{aligned}
$$

\section{Kalman Filtering based on ARMA Model}

For stationary random data containing colored noise, the kalman filter equation can not be used directly for compensation processing, otherwise there will be the situation of reduced filter accuracy or even distortion.Therefore, it is necessary to whiten the colored noise, Based on the relevant knowledge of the optimal state estimation, the state prediction value $\hat{X}_{k, k-1}$ and the observed prediction value $\tilde{Z}_{k, k-1}$ are respectively are the linear minimum variance estimates of $X_{k}$ and $Z_{k}$ obtained by the observation amount $Z^{k-1}$ at the previous n-1 time. According to the equivalence between the orthogonal projection theory and the minimum variance estimation, the kalman filter equation can be deduced under the colored noise using the orthogonal projection correlation property.

According to the previously determined ARMA $(3,1)$ model parameters, the system state equation and the measurement equation can be constructed:

$$
\left\{\begin{array}{l}
X_{k}=\Phi_{k, k-1} X_{k-1}+W_{k} \\
Z_{k}=H_{k} X_{k}+V_{k}
\end{array}\right.
$$

In the formula,

$X_{k}=\left[\begin{array}{l}x(k) \\ x(k-1) \\ x(k-2)\end{array}\right], \Phi_{k, k-1}=\left[\begin{array}{ccc}-a_{1} & -a_{2} & -a_{3} \\ 1 & 0 & 0 \\ 0 & 1 & 0\end{array}\right]$

$\Phi_{k, k-1}$ is a one-step transfer matrix, $Z(k)$ is the measured value, $H_{k}=\left[\begin{array}{lll}1 & 0 & 0\end{array}\right]$.

$W_{k}=\varepsilon(k)+b_{1} \varepsilon(k-1)$, among them, $\varepsilon(k)$ is the Gaussian white noise sequence, which is:

$$
\begin{aligned}
& E\left[\varepsilon(k) \varepsilon(k)^{T}\right]=\tilde{Q}_{k} \\
& \left.E] \varepsilon(k) \varepsilon(j)^{T}\right]=0, j \neq k
\end{aligned}
$$

Then

$$
\left\{\begin{aligned}
E\left[W_{k} W_{k}^{T}\right] & =E\left[\varepsilon(k) \varepsilon(k)^{T}\right]+b_{1}^{2} E\left[\varepsilon(k-1) \varepsilon(k-1)^{T}\right] \\
& =\tilde{Q}_{k}+b_{1}^{2} \tilde{Q}_{k-1}=Q_{k} \\
E\left[W_{k} W_{k-1}{ }^{T}\right] & =b_{1} E\left[\varepsilon(k-1) \varepsilon(k-1)^{T}\right]=b_{1} \tilde{Q}_{k-1} \\
& =M_{k} \\
E\left[W_{k} W_{k-j}{ }^{T}\right] & =0,|k-j| \geq 2
\end{aligned}\right.
$$

$V(k)$ is the system white noise:

$$
\begin{aligned}
& E\left[V_{k}\right]=0 \\
& E\left[V_{k} V_{k}^{T}\right]=R_{k} \\
& E\left[V_{k} V_{j}^{T}\right]=0, j \neq k
\end{aligned}
$$

Let $\Phi_{k, i} \Phi_{i, j}=\Phi_{k, j}, k>i>j$, From (5), it can be obtained: 


$$
\begin{aligned}
X_{k} & =\Phi_{k, k-1} X_{k-1}+W_{k} \\
& =\Phi_{k, 0} X_{0}+\sum_{i=1}^{k-1} \Phi_{k, i} W_{i}+W_{k} \\
Z_{k} & =H_{k} X_{k}+V_{k} \\
& =H_{k} \Phi_{k, 0} X_{0}+H_{k} \sum_{i=1}^{k-1} \Phi_{k, i} W_{i}+H_{k} W_{k}+V_{k}
\end{aligned}
$$

Innovation vector is:

$$
\tilde{e}_{k, k-1}=Z_{k}-H \hat{X}_{k, k-1}
$$

The Innovation covariance matrix is:

$$
E\left(\tilde{e}_{k} \tilde{e}_{k}^{T}\right)=H_{k} P_{k, k-1} H_{k}^{T}+R_{k}
$$

In the kalman filter equation, the state prediction $\hat{X}_{k, k-1}$ of step $k$ is the minimum variance estimate of the $X_{k}$ under the condition of the previous step $k-1$ measure $Z^{k-1}$, that is the orthogonal projection of $X_{k}$ in $Z^{k-1}$, Referred to as:

$$
\hat{X}_{k, k-1}=\operatorname{Pr}\left(X_{k} / Z^{k-1}\right)
$$

Substituting equation (5) into (13):

$$
\begin{aligned}
\hat{X}_{k, k-1} & =\Phi_{k, k-1} X_{k-1, k-1}+\operatorname{Pr}\left(W_{k} / Z^{k-1}\right) \\
& =\Phi_{k, k-1} X_{k-1, k-1}+\operatorname{Pr}\left(W_{k} /\left(Z^{k-2}, Z_{k-1}\right)\right)
\end{aligned}
$$

Then,

$\operatorname{Pr}\left(W_{k} /\left(Z^{k-2}, Z_{k-1}\right)\right)=\operatorname{Pr}\left(W_{k} / Z^{k-2}\right)+E\left[\tilde{W}_{k} \tilde{Z}_{k-1}^{T}\right]\left[E\left[\tilde{Z}_{k-1} \tilde{Z}_{k-1}^{T}\right]\right]^{-1} Z_{k-1}$

Where:

$$
\begin{aligned}
\tilde{W}_{k} & =W_{k}-\operatorname{Pr}\left(W_{k} / Z^{k-2}\right) \\
\tilde{Z}_{k-1} & =Z_{k-1}-\operatorname{Pr}\left(Z_{k-1} / Z^{k-2}\right) \\
& =Z_{k-1}-A_{k-1} \hat{X}_{k-1, k-2} \\
& =\tilde{e}_{k-1, k-2} \\
\operatorname{Pr} & \left(W_{k} / Z^{k-2}\right)=0
\end{aligned}
$$

And

$$
\begin{aligned}
E\left[\tilde{W}_{k} \tilde{Z}_{k-1}^{T}\right] & =E\left[\tilde{W}_{k}\left(Z_{k-1}-H_{k-1} \hat{X}_{k-1, k-2}\right)^{T}\right] \\
& =E\left[W_{k} Z^{T}{ }_{k-1}\right]+E\left[W_{k} X^{T}{ }_{k-1, k-2} H^{T}{ }_{k-1}\right) \\
& =M_{k} A^{T}{ }_{k-1}
\end{aligned}
$$

From (14)-(18), state prediction can be obtained:

$$
\hat{X}_{k, k-1}=\Phi_{k, k-1} X_{k-1, k-1}+M_{k} A^{T}{ }_{k-1}\left(H_{k} P_{k, k-1} H_{k}{ }^{T}+R_{k}\right) \tilde{e}_{k-1, k-2}
$$

Then one step prediction error:

$$
\breve{X}_{k, k-1}=\Phi_{k, k-1} \breve{X}_{k-1, k-1}+M_{k} A^{T}{ }_{k-1}\left(H_{k} P_{k, k-1} H_{k}{ }^{T}+R_{k}\right) \tilde{e}_{k-1, k-2}
$$

One step prediction covariance matrixcan can be getted:

$$
\begin{gathered}
P_{k, k-1}=E\left[\breve{X}_{k, k-1} \breve{X}_{k, k-1}^{T}\right]=\left[\begin{array}{c}
\Phi_{k, k-1} \\
I \\
-M_{k} A^{T}{ }_{k-1}\left(H_{k-1} P_{k-1, k-2} H_{k-1}{ }^{T}+R_{k-1}\right)^{-1}
\end{array}\right]^{T} \cdot \\
{\left[\begin{array}{ccc}
P_{k-1} & P_{1,2} & 0 \\
P^{T}{ }_{1,2} & Q_{k} & P_{2,3} \\
0 & P^{T}{ }_{2,3} & H_{k} P_{k, k-1} H_{k}{ }^{T}+R_{k}
\end{array}\right]\left[\begin{array}{c}
{ }_{k, k-1} \\
I \\
-\left(H_{k-1} P_{k-1, k-2} H_{k-1}{ }^{T}+R_{k-1}\right)^{-1} A_{k-1} M^{T}{ }_{k}
\end{array}\right]}
\end{gathered}
$$

Among them, $P_{1,2}=E\left[\breve{X}_{k-1, k-1} W_{k}^{T}\right]=\left(I-K_{k-1} A_{k-1}\right) N_{k}{ }^{T} \quad P_{2,3}=E\left[W_{k} \tilde{e}_{k-1, k-2}\right]=N_{k} A_{k-1}{ }^{T}$.

From equation (21), we can get the state prediction value of colored noises and one more control item. This is made up of the previous message vector and gain matrix, The covariance matrix also contains information about the colored noise, which visually shows the influence of the colored noise in the kalman filtering process and is controlled. The improved filtering equation is as follows: 
State step prediction: as shown in equation (19),

State Estimation:

$$
\hat{X}_{k}=\hat{X}_{k, k-1}+K_{k} \tilde{e}_{k, k-1}
$$

Filter gain matrix:

$$
K_{k}=P_{k, k-1} H_{k}^{T}\left[\frac{1-\rho^{k}}{1-\rho}\left(H_{k} P_{k, k-1} H_{k}^{T}+R_{k}^{T}\right)\right]^{-1}
$$

In the $K_{k}$ equation, the variable weighting coefficient $\frac{1-\rho^{k}}{1-\rho}$ is introduced, and on the one hand, $P_{k, k-1}$ can be adjusted to enhance the effect of the new measurement. On the other hand, the noise covariance matrix is adjusted to ensure the stability of the covariance matrix Sex, and then improve the filtering accuracy.

Estimated Filtering Variance Matrix:

$$
P_{k}=\left(I-K_{k} A_{k}\right) P_{k, k-1}\left(I-K_{k} A_{k}\right)^{T}+K_{k} R_{k} K_{k}^{T}
$$

System noise variance matrix:

$$
\hat{Q}_{k}=\left(1-\frac{1}{k}\right) \hat{Q}_{k-1}+\frac{1}{k}\left(K_{k} e_{k, k-1} e_{k, k-1}{ }^{T} K_{k}^{T}+P_{k}-H_{k} P_{k-1} H_{k}^{T}\right)
$$

The initial value is:

$$
Q_{0}=\frac{1}{N-n} \sum_{k=n+1}^{N} \xi(k)^{T} \xi(k)\left[\begin{array}{ccc}
1 & 0 & 0 \\
0 & \ddots & 0 \\
0 & 0 & 1
\end{array}\right]
$$

$\xi(k)$ is the residual sequence fitted by the ARMA model. Measurement noise variance matrix:

$$
R=\frac{1}{N} \sum_{k=1}^{N}[r z(k)]^{2}
$$

Where $r$ is the percentage of the data assumed to be the model error, Set to 0.1. According to equations (19),(22) to (27), kalman filtering under colored noise conditions can be performed.So as to obtain the optimal estimate of HRG stationary random data.

\section{Data Validation and Effect Analysis}

According to the HRG stationary random signal of Fig.1, the ARMA model of equation (4) is established, and then the state and measurement equations are established, and then the classical kalman filter and the new kalman filter method are used to compensate. As shown in Fig 4 and Fig 5.

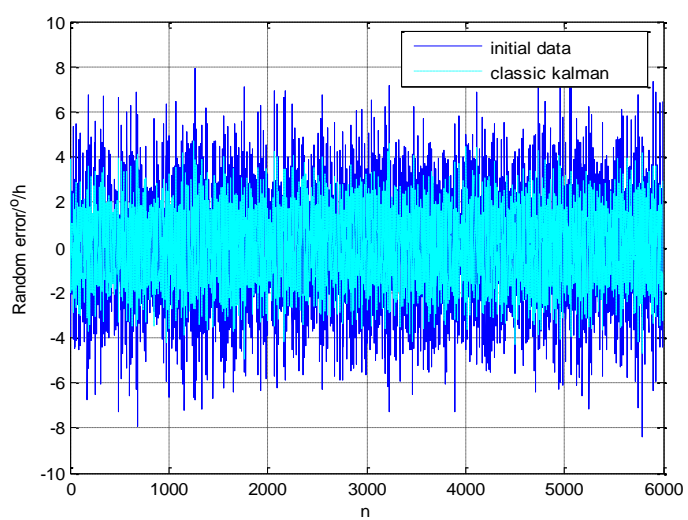

Figure 4. Classic kalman filter results

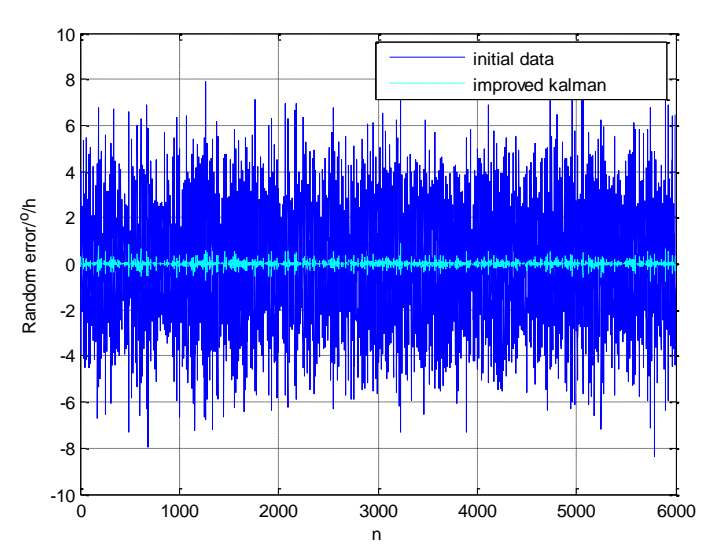

Figure 5. Improved kalman filter results

It can be clearly seen from Fig. 4 and Fig. 5 that after the classical kalman filtering, the fluctuation range of the data is slightly reduced, and after the new method, the fluctuation of the data changes obviously. In order to more clearly quantitative analysis smooth random signal, the classic kalman filtered signal, the improved method of this paper after the statistical characteristics of the statistical comparison shown in Table 1. 
Table1. The statistical feature analysis of random error

\begin{tabular}{|c|c|c|c|}
\hline & $\begin{array}{c}\text { Smooth Random } \\
\text { Signal }\end{array}$ & $\begin{array}{c}\text { The Classic Kalman } \\
\text { Filter }\end{array}$ & The Improved Method \\
\hline Mean $\left({ }^{\circ} / h\right)$ & $1.710 \mathrm{e}-04$ & 3.725 e-04 & $4.805 \mathrm{e}-05$ \\
\hline Variance ${ }^{\left((\circ / h)^{2}\right)}$ & 5.812 & 2.017 & $8.531 \mathrm{e}-03$ \\
\hline
\end{tabular}

From the data in the table, it can be seen that the classical filtering algorithm has little improvement in the filtering effect due to the absence of colored noise, and the variance is slightly reduced;however, the improved kalman filter has obvious improvement in the characteristics of the filtered data. The random signal is reduced by three orders of magnitude, indicating that the discrete characteristics have been improved obviously, and the improved filtering algorithm is also verified indirectly.

In order to further illustrate the effectiveness of the improved filtering algorithm, the classical kalman stationary random signal and the improved filtered signal are analyzed by Allan variance, as shown in Fig.6, Fig.7 and Table 2.

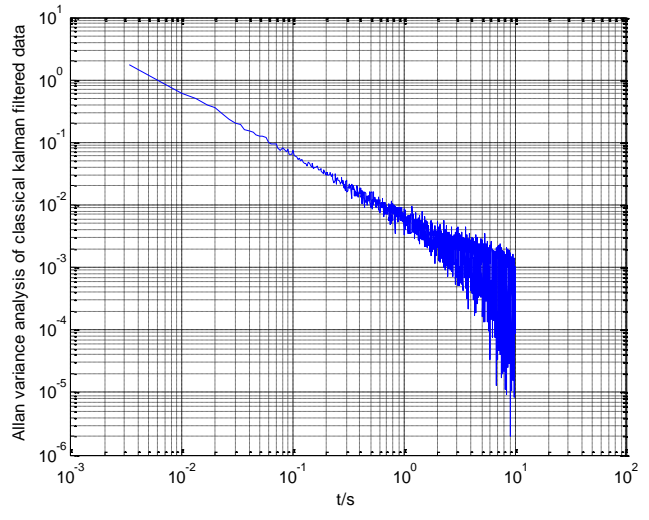

Figure 6 classical kalman filtered data

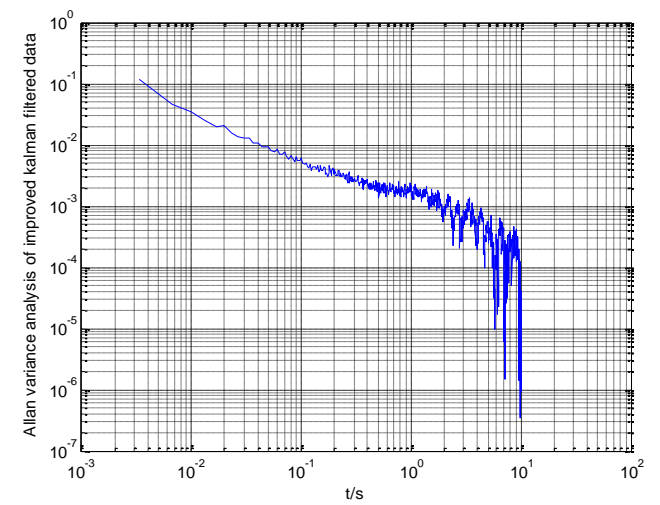

Figure 7. Improved kalmanfiltered data

Table 2. The identification result contrast of HRG random error

\begin{tabular}{ccccc}
\hline $\begin{array}{c}\text { The main error } \\
\text { coefficients }\end{array}$ & $\begin{array}{c}\text { Smooth } \\
\text { Random Signal }\end{array}$ & $\begin{array}{c}\text { The Classic } \\
\text { Kalman Filter }\end{array}$ & $\begin{array}{c}\text { The Improved } \\
\text { Method }\end{array}$ & Rate of change \\
\hline $\begin{array}{c}\text { Quantization Noise } \\
\text { Coefficient } /(\text { urad })\end{array}$ & 117.01 & 53.194 & 4.2969 & $-96.33 \%$ \\
$\begin{array}{c}\text { Angle Random Walk } \\
\text { Coefficient } /\left(\left(^{\circ}\right) / h^{1 / 2}\right)\end{array}$ & 1.8678 & 0.57917 & 0.06427 & $-96.59 \%$ \\
$\begin{array}{c}\text { Rate Slope } \\
\text { Coefficient } /\left(\left(^{\circ}\right) / h^{2}\right) \\
\text { Rate Travel } \\
\text { Coefficient } \\
\left./\left({ }^{\circ}\right) / h^{3 / 2}\right)\end{array}$ & $1.2544 \mathrm{e}+04$ & $3.9291+04$ & $8.9988 \mathrm{e}+03$ & $-28.26 \%$ \\
$\begin{array}{c}\text { Zero Partial } \\
\text { Instability }\end{array}$ & 13906 & 4365 & 1079.5 & $-92.24 \%$ \\
Coefficient $/\left(\left(^{\circ}\right) / h\right)$ & 328.44 & 104.54 & 25.669 & $-92.18 \%$ \\
\hline
\end{tabular}

The table "-" represents the reduction of the modified algorithm compared to the original stochastic steady data.

From the data analysis in the table, we can see that after the classical kalman filtering, most of the error term coefficients are obviously reduced, but the value of the slope coefficient increases, which means that the effect of kalman filter appears when considering colored noise divergent phenomenon. After improved kalman filtering, the data of the five error terms are significantly reduced. This shows that the error characteristics of HRG stationary random data are improved well, and the superiority of improved filtering method is also verified. 


\section{Conclusion}

This paper mainly studies the HRG stochastic error modeling and filtering method based on ARMA model, which has certain application value to reduce the random error of gyroscope. The main results are as follows: (1) The idea and main method of ARMA model modeling are given; (2) Using the orthogonal projection principle, a new method of whitening the colored noise is deduced, and the influence of colored noise on the system is displayed intuitively; (3) The kalman filter equation under colored noise is established, and the filtering effect of the improved algorithm and the classical algorithm is compared. The modeling accuracy and good filtering effect of the gyroscope smooth random data are obtained.

\section{Acknowledgments}

Fund Project: National Natual Science Foundation(61174030);

About the author:YANG Haotian,(1991-), male, Xi'an, shaanxi, Ph.D, is mainly engaged in HRG error modeling analysis.

\section{References}

[1]. Guo Ling, Wu Xun-zhong, Jin Yi. Building model of the drift of the fiber optic gyroscope and application in the error equation of inertial navigation system[J].Optical Techique, Vol. 39 (2013) No. 4, p.328-330.

[2]. Du HongSong, Cheng Jian Hong, Tang Miao Miao. Stochastic error modeling method for micro inertial sensor based on ARMA[J]. Transducer and Microsystem Technologies, Vol.32 (2013) No.4, p. 54-64.

[3]. Qian Huaming, et al. Smiulation and expermient of random errors of MEMS gyroscope [J]. Journal of Beijing University of Aeronautics and Astronautics, Vol. 36 (2010) No. 6, p. 636-639.

[4]. Zhang Chun-xi, Wang Lu, Wang Zhen-fei. RLG random error modeling method in initial alignment application [J]. Infrared and Laser Engineering, Vol. 42(2013) No. 1, p.62-67.

OLi Jian li, Jiao Feng, Fang JiangCheng, et al. Temperature error modeling of RLG based on neural network optimized by PSO and regularization [J]. IEEE Sensors Journal, Vol. 14 (2014) No. 3, p.:912-919.

[5]. Diao Zhang lin, Quan HaiYang, Lan LiDong, et al. Analysis and compensation of MEMS gyroscope drift[C]//Proceedings of 2013 IEEE Seventh International Conference on Sensing Technology.Piscataway, NJ: IEEE Press, 2013, p. 592-596.

[6]. Chen Fang, Wu Xiaobei, Liu Wenyi etc. Kalman filter under colored noise [J]. Journal of Xi'an University of Posts and Telecommunications, Vol. 19 (2013) No. 2, p.56-59.

[7]. Song Guodong, Jiang Shouda, Lin Lenglei. Information fusion Kalman filter for complex colored noise singular systems [J]. Journal of Instrumentation,Vol. 34(2013) No. 5, p.1195-1120.

[8]. Zeng Qing-hua, Huang Lei, Liu Jian-ye et al. Real-time filtering methods of FOG random noise based on ARMA model[J].Journal of Chinese Inertial Technology, Vol. 23 (2013) No. 1, p.120124.

[9]. Kim D, M'Ccloskey R T. Spectral analysis of vibratory gyro noise [J].Sensors Journal, IEEE, Vol. 13 (2013) No.11, p.4361-4374.

[10]. Lei H.Auto Regressive Moving Average (ARMA) Modeling Method for Gyro Random Noise Using a Robust Kalman Filter [J]. Sensors Journal, IEEE,Vol. 15 (2013) No. 1, p.25277-25286.

[11]. Yan Gongmin, Li Sihai, Qin Yongyuan. Inertia Instrument Testing in Data Analysis [M]. Beijing: Defense Industry Press, 2012.p.28-30.

[12]. Lloyd S W, Digonnet M J F, Fan Shan-hui.Modeling coherent backscattering errors in fiber optic gyroscopes for sources of arbitrary line width [J]. Lightwave Technology, Vol. 31 (2013) No. 13, p.2070-2078. 